\title{
Nonlinear Soil Response via Harmonic-Modal Hybrid Approach
}

\author{
Claudia Germoso ${ }^{1}$, Francisco Chinesta ${ }^{2}$, Juan Aracena ${ }^{1}$ \\ ${ }^{1}$ Instituto Tecnológico de Santo Domingo (INTEC) \\ Av. Los Próceres, Jardines del Norte, Santo Domingo, República Dominicana \\ Claudia.germoso@intec.edu.do; juanaracena09@gmail.com \\ ${ }^{2}$ ESI GROUP Chair @ PIMM, Arts et Métiers Institute of Technology, \\ 151, boulevard de l'Hôpital, 75013 Paris, France \\ Francisco.Chinesta@esi-group.com
}

\begin{abstract}
Non-linear behaviour of soils during a seismic event has a predominant role in current site response analysis. Soil response analysis consistently indicates that the stress-strain relationship of soils is non-linear and shows hysteresis. An equivalent linearization method, in which non-linear characteristics of shear modulus and damping factor of soils are modeled as equivalent linear relations of the shear strain is usually applied, but this assumption, however, may lead to a conservative approach of the seismic design. In this paper, we propose a new harmonic analysis formulation, able to address forced response simulation of soils exhibiting their characteristic nonlinear behavior. A possibility for represent this non-linear analysis consist in combining modal and harmonic analysis for defining an hybrid integration scheme.
\end{abstract}

Keywords: Nonlinear soil behavior, Proper Generalized Decomposition, Harmonic analysis, Modal analysis, dynamics

\section{Introduction}

Solid dynamics is usually formulated either in the time or in the frequency domains. The first is preferred when calculating transient responses, whereas the frequency approach is an appealing alternative for calculating forced responses, both extensively used and described in many reference books, as for example [4]. The general discrete form of linear solid dynamics writes

$$
\mathbf{M} \frac{d^{2} \boldsymbol{U}(t)}{d t^{2}}+\mathbf{C} \frac{d \boldsymbol{U}(t)}{d t}+\mathbf{K} \mathbf{U}(\mathrm{t})=\mathbf{F}(\mathrm{t})
$$

where $\mathbf{M}, \mathbf{C}$, and $\mathbf{K}$ are respectively the mass, damping and stiffness matrices, $\mathbf{U}$ the vector that contains the nodal displacements and $\mathbf{F}$ the nodal excitations (forces).

The main drawback related to the time integration of Eq. (1) lies in the necessity of solving a linear system (usually of very large size) at each time step, in particular when some of these matrices change in time for a variety of reasons (time dependent behavior, nonlinearities, ...).

Loads can be easily expressed in the frequency domain. In what follows we consider without loss of generality the simplest scenario: $\mathbf{F}(\mathrm{t})=\mathbf{f} g(t)$, with $\|\mathbf{f}\|=1$. The time function $g(t)$ can be expressed from the superposition of harmonic functions $e^{i \omega t}$ with $\omega$ the circular frequency and $i=\sqrt{-1}$. If we assume a single frequency harmonic excitation $(t)=e^{i \omega t}$ , the response of a linear solid is expected having the same frequency but exhibiting a certain phase angle $\theta$, i.e. $\mathbf{U}(\mathrm{t})=$ $\overline{\mathbf{U}} e^{i \omega t+i \theta}$, where is the $\overline{\mathbf{U}}$ the vector containing the amplitude of the nodal displacements. This vector can be rewritten as $\mathbf{U}(\mathrm{t})=\overline{\mathbf{U}} e^{i \omega t+i \theta}=\mathbb{U} e^{i \omega t}$, where now $\mathbb{U}=\overline{\mathbf{U}} e^{i \theta}$ denotes a vector of complex entries, with $\mathbb{U}=\mathbf{U}_{\mathbf{r}}+\mathbf{i} \mathbf{U}_{\mathbf{i}}$, where $\mathbf{U}_{\mathbf{r}}$ and $\mathbf{U}_{\mathbf{i}}$ are respectively the real and imaginary parts of $\mathbb{U}$.

By introducing $\mathbf{F}(\mathrm{t})=\mathbf{f} e^{i \omega t}$ and $\mathbf{U}(\mathrm{t})=\mathbb{U} e^{i \omega t} \mathrm{t}$ into Eq. (1) results the frequency-based description of solid dynamics

$$
\left(-\omega^{2} \mathbf{M}+i \omega \mathbf{C}+\mathbf{K}\right) \mathbb{U}=\mathbf{f}
$$


where the exponential factor $e^{i \omega t}$ was eliminated from both members. Imagine for a while that damping vanishes, i.e. $\mathbf{C}=0$, and that we focuses on the free response of the mechanical system, i.e. $\mathbf{f}=\mathbf{0}$. In this case Eq. (2) reduces to:

$$
\mathbf{K} \mathbb{U}=\omega^{2} \mathbf{M} \mathbb{U}
$$

that defines an eigenproblem that results in the eigenmodes $\mathbf{P}_{i}$ and the associated eigenfrequencies $\omega^{2}$. The inverse transform allows coming back to the time domain through the Fourier transform $\mathcal{F}(\cdot), \mathbf{U}(\mathrm{t})=\mathcal{F}^{-1}(\mathbb{U}(\omega))$.

\section{Nonlinear soil behavior}

It is well established in geotechnical engineering that soil response is nonlinear beyond a certain level of deformation. Stress-strain relationships for the levels of shearing deformation produced by large earthquakes are nonlinear and hysteretic, as has been confirmed by numerous results of vibratory and cyclic loading tests on soil samples.

The performance of cyclic nonlinear models can be illustrated by a very simple example in which the shape of the backbone curve is described by $\tau=f(\gamma)$. The shape of any backbone curve is tied to two parameters, the initial (low strain) stiffness and the (high-strain) shear strength of the soil [8].

Of hyperbolic type models, the most famous and most widely used model is the one initially proposed by [9] (referred to as KZ "Kondner and Zelasko" model hereafter) and lately redefined by [6]. Kondner and Zelasko [9] formulated the stress-strain relationship for skeleton curves by the hyperbolic equation as follows,

$$
\tau=f(\gamma)=\frac{G \gamma}{1+\frac{G}{\tau_{\max }}|\gamma|}
$$

where $\tau$ is the shear stress, $\gamma$ is the shear strain, $G$ is the undisturbed shear modulus (taken at the origin) and $\tau_{\max }$ is the shear strength (the maximum stress that material can support in the initial state, $\tau_{\max }=G \gamma_{r}$. In the numerical test here addressed, it is assumed that

$$
\tau=\mathrm{G}\left(\gamma+c \frac{\gamma}{1+\frac{1}{\gamma_{r}}|\gamma|}\right)
$$

where $\gamma_{r}$ is the reference deformation and $\mathrm{c}$ can be considered as a parameters (let's note that when $\mathrm{c}=0$ the nonlinear case reduces to the linear case).

The other parameter is the damping characteristics of the soil (represented by the damping ratio $\zeta$ ) which is calculated by the ratio of the energy dissipated $W_{D}$ and energy of elastic deformation $W_{E}$ from the hysteretic loop, i.e.

$$
\zeta=\frac{W_{D}}{4 \pi W_{E}}
$$

Ishihara's research [7] indicated that by applying Masing's Rule to hyperbolic type initial loading curves, the damping ratio defined by Eq.(6) can be computed as

$$
\zeta=\frac{2}{\pi}\left(\frac{2 \int_{0}^{\gamma_{a}} f(\gamma) d \gamma}{f\left(\gamma_{a}\right) \gamma_{a}}-1\right)
$$

where $\gamma_{a}$ is the angular deformation. Applying the hyperbolic equation given by Eq. (4) the damping ratio $(\zeta)$ is reached 


$$
\zeta=\frac{4}{\pi}\left(1+\frac{1}{\frac{\gamma_{a}}{\gamma_{r}}}\right)\left[1-\frac{\ln \left(1+\frac{\gamma_{a}}{\gamma_{r}}\right)}{\frac{\gamma_{a}}{\gamma_{r}}}\right]-\frac{2}{\pi}
$$

\section{Harmonic-Modal Hybrid Approach}

This section describe a Hybrid technique which provide a specially suitable method based on modal and harmonic analysis [11][13]. We now consider matrix $\mathbf{P}$ diagonalizing matrices $\mathbf{M}, \mathbf{C}$ and $\mathbf{K}$ from the Eq. (2), that is

$$
\begin{aligned}
& \mathbf{P}^{\mathrm{T}} \mathbf{M} \mathbf{P}=\mathbb{M} \\
& \mathbf{P}^{\mathrm{T}} \mathbf{K} \mathbf{P}=\mathbb{K} \\
& \mathbf{P}^{\mathrm{T}} \mathbf{C} \mathbf{P}=\mathbb{C}
\end{aligned}
$$

with $\mathbb{M}_{i j}=m_{i i} \delta_{i j}, \mathbb{K}_{i j}=k_{i i} \delta_{i j}, \mathbb{C}_{i j}=c_{i i} \delta_{i j}$, with $\delta_{i j}$, the Kroenecker's delta, i.e. $\mathbb{M}, \mathbb{K}$ and $\mathbb{C}$ becomes diagonal with entries $m_{i i}, c_{i i}$, and $k_{i i}$ respectively. Such a choice implies that the system is no longer described in terms of its nodal degrees of freedom but rather in terms of the modal content.

The two are formally related through the linear transformation

$$
\mathbb{U}(\omega)=\mathbf{P} \xi(\omega)
$$

Thus, the dynamical problem reduces to

$$
\left(-\omega^{2} \mathbb{M}+i \omega \mathbb{C}+\mathbb{K}\right) \xi(\omega)=\mathbf{P}^{\mathbf{T}} \mathbf{F}(\boldsymbol{\omega})=\mathbb{f}(\boldsymbol{\omega})
$$

that result in a system of $N_{n}$ decoupled algebraic equations ( $N_{n}$ being the size of matrices $\mathbf{M}, \mathbf{C}$ and $\left.\mathbf{K}\right)$

$$
\left(-\omega^{2} m_{i i}+i \omega c_{i i}+k_{i i}\right) \xi_{i}(\omega)=f_{i}(\omega)
$$

with $i=1,2, \ldots N_{n}$. From which it results

$$
\xi_{i}(\omega)=\frac{f_{i}(\omega)}{\left(-\omega^{2} m_{i i}+i \omega c_{i i}+k_{i i}\right)}
$$

that allows calculating the nodal amplitudes from Eq. (10), i.e. $\mathbb{U}(\omega)=\mathbf{P} \xi(\omega)$. Thus, the space-frequency separated representation reads

$$
\mathbb{U}(\omega)=\sum_{i=1}^{N_{n}} \mathbf{Z}_{\mathbf{i}} \xi_{\mathrm{i}}(\omega)
$$

where $\mathbf{Z}_{\mathbf{i}}$ is the $\mathrm{i}$-column of matrix $\mathbf{P}$. The obtention of $\mathbb{U}(\omega)$ allows us to come back to the time domain $\mathrm{U}(\mathrm{t})$ by applying an inverse Fourier transform,

$$
\mathbf{U}(\mathrm{t})=\mathcal{F}^{-1}(\mathbb{U}(\omega))
$$

\section{Nonlinear dynamics}

In the nonlinear case, the general semi discretized equilibrium dynamic equation writes 


$$
\mathbf{M} \frac{d^{2} \boldsymbol{U}(t)}{d t^{2}}+\mathbf{C} \frac{d \boldsymbol{U}(t)}{d t}+\mathbf{K} \mathbf{U}(\mathrm{t})-\mathbf{H}(\mathbf{U})=\mathbf{F}(\mathrm{t})
$$

where the nonlinear contribution is grouped in the vector $\mathbf{H}(\mathbf{U})$. Now, the simplest linearization consists of looking for the solution $\mathbf{U}(\mathrm{t})$ from the nonlinear term taken at the previous iteration that for the sake of notational simplicity is denoted by $\mathbf{U}^{-}(\mathrm{t})$. Thus, as soon as the nonlinear contribution is assumed, it can be moved to the right hand member, i.e.

$$
\mathbf{M} \frac{d^{2} \boldsymbol{U}(t)}{d t^{2}}+\mathbf{C} \frac{d \boldsymbol{U}(t)}{d t}+\mathbf{K} \mathbf{U}(\mathrm{t})=\mathbf{c H}\left(\mathbf{U}^{-}(\mathrm{t})\right)+\mathbf{F}(\mathrm{t})
$$

and at present iteration the damping ratio can be updated from the Eq. 3. Now, a direct possibility consists in computing the Fourier transform of the right-hand member

$$
\mathbf{F}(\omega)=\mathcal{F}\left(\mathbf{H}\left(\mathbf{U}^{-}(\mathrm{t})\right)+\mathbf{F}(\mathrm{t})\right)
$$

and then to proceed exactly in the same way than in the linear case. However, in order to take benefit from model order reduction, in what follow we present an alternative but equivalent formulation, more adapted to the use of reduced bases. By invoking the linearity of Fourier transform, we write

$$
\mathbf{F}(\omega)=\mathcal{F}\left(\mathbf{H}\left(\mathbf{U}^{-}(\mathrm{t})\right)+\mathbf{F}(\mathrm{t})\right)=\mathcal{F}\left(\mathbf{H}\left(\mathbf{U}^{-}(\mathrm{t})\right)\right)+\mathcal{F}(\mathbf{F}(\mathrm{t}))=\mathbf{F}^{H}(\omega)+\mathbf{F}^{F}(\omega)
$$

which could be expressed using a pricewise linear approximation basis $N_{l}(\omega)$ (like the usually considered one in linear finite element analyses)

$$
\mathbf{F}^{H}(\omega)=\sum_{l=1}^{L} \mathbf{F}^{H}\left(\omega_{l}\right) N_{l}(\omega) \rightarrow \mathbb{f}^{H}(\omega)=\mathbf{P}^{\mathbf{T}} \mathbf{F}^{\mathbf{H}}(\omega)
$$

and

$$
\mathbf{F}^{F}(\omega)=\sum_{l=1}^{L} \mathbf{F}^{F}\left(\omega_{l}\right) N_{l}(\omega) \rightarrow \mathbb{f}^{F}(\omega)=\mathbf{P}^{\mathbf{T}} \mathbf{F}^{\mathbf{F}}(\boldsymbol{\omega})
$$

each one contribution to the solution $\xi(\omega)$ according to

$$
\xi_{i}(\omega)=\xi^{\mathrm{H}}(\omega)+\xi^{\mathrm{F}}(\omega)
$$

with

$$
\begin{aligned}
& \xi_{i}^{H}(\omega)=\frac{\sum_{l=1}^{L} \mathbb{f}_{i}^{H}(\omega) N_{l}(\omega)}{\left(-\omega^{2} m_{i i}+i \omega c_{i i}+k_{i i}\right)}, \quad i=1,2, \ldots, N_{n} \\
& \xi_{i}^{F}(\omega)=\frac{\sum_{l=1}^{L} \mathbb{f}_{i}^{F}(\omega) N_{l}(\omega)}{\left(-\omega^{2} m_{i i}+i \omega c_{i i}+k_{i i}\right)}, \quad i=1,2, \ldots, N_{n}
\end{aligned}
$$


Thus, it finally results

$$
\mathbb{U}(\omega)=\mathbf{P} \xi(\omega)=\mathbb{U}^{\mathrm{H}}(\omega)+\mathbb{U}^{\mathrm{F}}(\omega)=\mathbf{P} \xi^{\mathrm{H}}(\omega)+\mathbf{P} \xi^{\mathrm{F}}(\omega)
$$

and coming back to the time domain

$$
\mathbf{U}(\mathrm{t})=\mathbf{U}^{\mathbf{H}}(\mathrm{t})+\mathbf{U}^{\mathbf{F}}(\mathrm{t})=\mathcal{F}^{-1}\left(\mathbb{U}^{\mathbf{H}}(\omega)\right)+\mathcal{F}^{-1}\left(\mathbb{U}^{\mathbf{F}}(\omega)\right)
$$

\section{Separated representation of nonlinear soil dynamics}

In this section, we describe the construction of the parametric solutions within the Proper Generalized Decomposition framework, largely considered in our former works [1] [2] [3] [12]. In the nonlinear case, damping is considered as a

parameter within the PGD framework in order to update regarding the level of deformation. For this purpose, we consider first the separated representation construction of the single-stratum parametric displacement

where

$$
\mathbb{U}\left(z, \omega, \zeta_{1}, \ldots, \zeta_{n}\right)=\sum_{i=1}^{N_{n}} \mathbf{Z}_{\mathbf{i}} \zeta_{\mathrm{i}}\left(z, \omega, \zeta_{1}, \ldots, \zeta_{n}\right)
$$

$$
\xi_{i}\left(z, \omega, \zeta_{1}, \ldots, \zeta_{n}\right) \approx \sum_{k=1}^{m} \mathrm{X}_{\mathrm{k}}(z) \mathrm{W}_{\mathrm{k}}(\omega) \mathcal{M}_{\mathrm{k}}^{1}\left(\zeta_{1}\right) \ldots \mathcal{M}_{\mathrm{k}}^{\mathrm{n}}\left(\zeta_{n}\right)
$$

with $n=1,2 \ldots L$ ( $L$ soil layers). The PGD constructor can be easily implemented to the harmonic equation (10) given by

$$
\left(-\omega^{2} \mathbb{M}+i \omega \mathbb{C}+\mathbb{K}\right) \xi(\omega)=\mathbb{f}(\boldsymbol{\omega})
$$

with $\mathbb{f}(\boldsymbol{\omega})=\mathbb{f}^{H}(\omega)+\mathbb{f}^{F}(\omega)$. Now, considering the weighted residual form

$$
\int_{\Omega} \xi^{* T}\left(-\omega^{2} \mathbb{M}+i \omega \mathbb{C}+\mathbb{K}\right) \xi=\int_{\Omega} \xi^{*} \mathbb{f}
$$

where $\Omega=\Omega_{\mathrm{z}} \times \Omega_{\omega} \times \Omega_{\zeta_{1}} \ldots \times \Omega_{\zeta_{n}}$. The PGD approximation sought the form

$$
\xi_{i}^{m}(z, \omega, \zeta) \approx \sum_{k=1}^{\boldsymbol{m}-\mathbf{1}} X_{k}(z) W_{k}(\omega) \mathcal{M}_{k}^{1}\left(\zeta_{1}\right) \ldots \mathcal{M}_{k}^{n}\left(\zeta_{n}\right)+X_{m}(z) W_{m}(\omega) \mathcal{M}_{m}^{1}\left(\zeta_{1}\right) \ldots \mathcal{M}_{m}^{n}\left(\zeta_{n}\right)
$$

The introduction of Eq. (28) into to (27) results a non-linear problem. We proceed by considering the simplest linearization strategy, an alternated directions fixed point algorithm. Assuming $\xi^{m-1}$ to be known, we can compute the next iteration $\xi^{m}$. Similarly we suppose sequentially that $W_{m}(\omega)$, and $\mathcal{M}_{m}^{1}\left(\zeta_{1}\right) \ldots \mathcal{M}_{m}^{n}\left(\zeta_{n}\right)$ are known of the previously iteration and proceed to compute $X_{m}(z)$.With the new value of $X_{m}(z)$ and $\mathcal{M}_{m}^{1}\left(\zeta_{1}\right) \ldots \mathcal{M}_{m}^{n}\left(\zeta_{n}\right)$ previously assumed, we can obtain $W_{m}(\omega)$, the process continue until reach $\mathcal{M}_{m}^{1}\left(\zeta_{1}\right) \ldots \mathcal{M}_{m}^{n}\left(\zeta_{n}\right)$ sequentially and so on until reaching a state of convergences, where the results will be the new products $X_{m}(z), W_{m}(z)$ and $\mathcal{M}_{m}^{n}(z)$. The enrichment stop when the model residual become small enough. 


\section{Numerical Example}

In this section, a numerical example for illustrating the potentialities of the technique just proposed are presented. The PGD approach was deeply compared with the solutions obtained by using the DEEPSoil software. We consider a soil deposit consisting of 5 stratums. The PGD method was used to calculate the parametric solution to the displacement field $\xi\left(z, \omega, \zeta_{1}, \zeta_{2}, \zeta_{3}, \zeta_{4}, \zeta_{5}\right)$. The problem's coordinates are defined in the domains $=(0,7.5), \omega=2 \pi(0,25) s-1$, and $\zeta=$ $(0.01,0.5)$. The different domains were discretized by considering respectively 100, 1023 and 100 nodes. Even if 100 nodes for discretizing the parametric domains seem too much, as the calculation of functions depending on the parameters does not imply the solution to linear systems, it is preferable to consider a rich-enough discretization to be sure of representing accurately the parametric solution. In the numerical simulation carried out and discussed below we consider 5 layers and a half-space with the properties in Table 1 . In the present case, the damping contribution was modeled considering a viscosity $\left(\eta=\frac{2 \zeta}{\omega}\right)$ from

which lead to

$$
\eta \frac{d \boldsymbol{U}(t)}{d t}=2 \mathrm{i} \zeta \boldsymbol{U}(t)
$$

$$
\mathbf{C}=2 \mathrm{i} \zeta \mathbf{K}
$$

Table 1: Material parameters.

\begin{tabular}{|c|c|c|c|c|c|}
\hline Stratum & Thickness (m) & Shear Modulus (Pa) & Damping (\%) & Density $\left(\mathbf{k g} / \mathbf{m}^{\mathbf{3}}\right)$ & $\boldsymbol{\gamma}_{\boldsymbol{r}}(\boldsymbol{\%})$ \\
\hline 1 & 1.5 & 15657000 & $1 \%$ & 1940 & 0.1280 \\
\hline 2 & 2 & 22791000 & $1 \%$ & 1940 & 0.1280 \\
\hline 3 & 2 & 26861000 & $1 \%$ & 1940 & 0.1630 \\
\hline 4 & 1.3 & 31266000 & $1 \%$ & 1940 & 0.1630 \\
\hline 5 & 0.7 & 46492000 & $1 \%$ & 1940 & 0.1630 \\
\hline Half-space & & 3336000000 & $0 \%$ & 2200 & - \\
\hline
\end{tabular}

In soil analysis, the control is defined from the response spectrum, or its corresponding time history, at bedrock by what is known as rock outcropping motion.

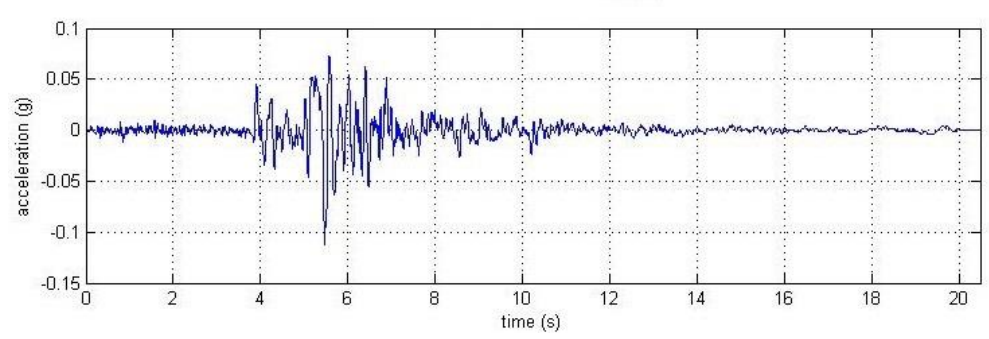

Fig. 1: Seismic loading.

From the outcropping motion, the objective is to predict the bedrock motion covered by the soil deposit. Thus, the bedrock half-space can be substituted with boundary condition

$$
\tau_{\mathrm{o}}^{*}=c_{s^{*}} \rho_{b} A \dot{U}_{s}-c_{s^{*}} A \rho_{b} \dot{U}_{o}^{*}
$$


where $\dot{U}_{S}$ the rock outcropping velocity (assumed measurable) and $\dot{U}_{o}^{*}$ the velocity at the base of the soil column [5][10], that coincides with the soil-bedrock half-space interface and $\tau_{o}^{*}$ the shear stress at that position. The solution is obtained assuming we know the acceleration time history in the outcropping, shown in Fig. 1.
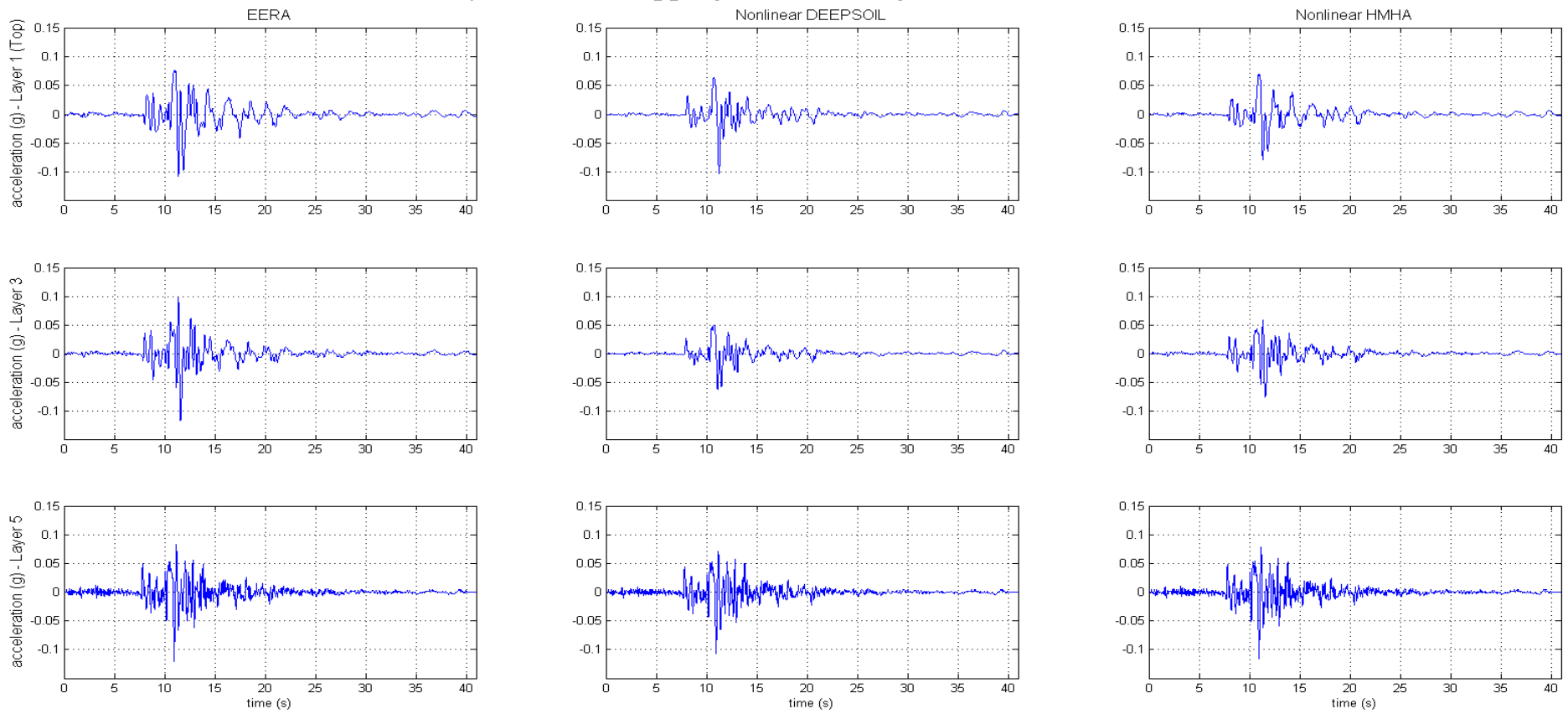

Fig. 2: Acceleration time history of layer 1, layer 3 and layer 5 for the EERA, DEEPSOIL and NHMHA.
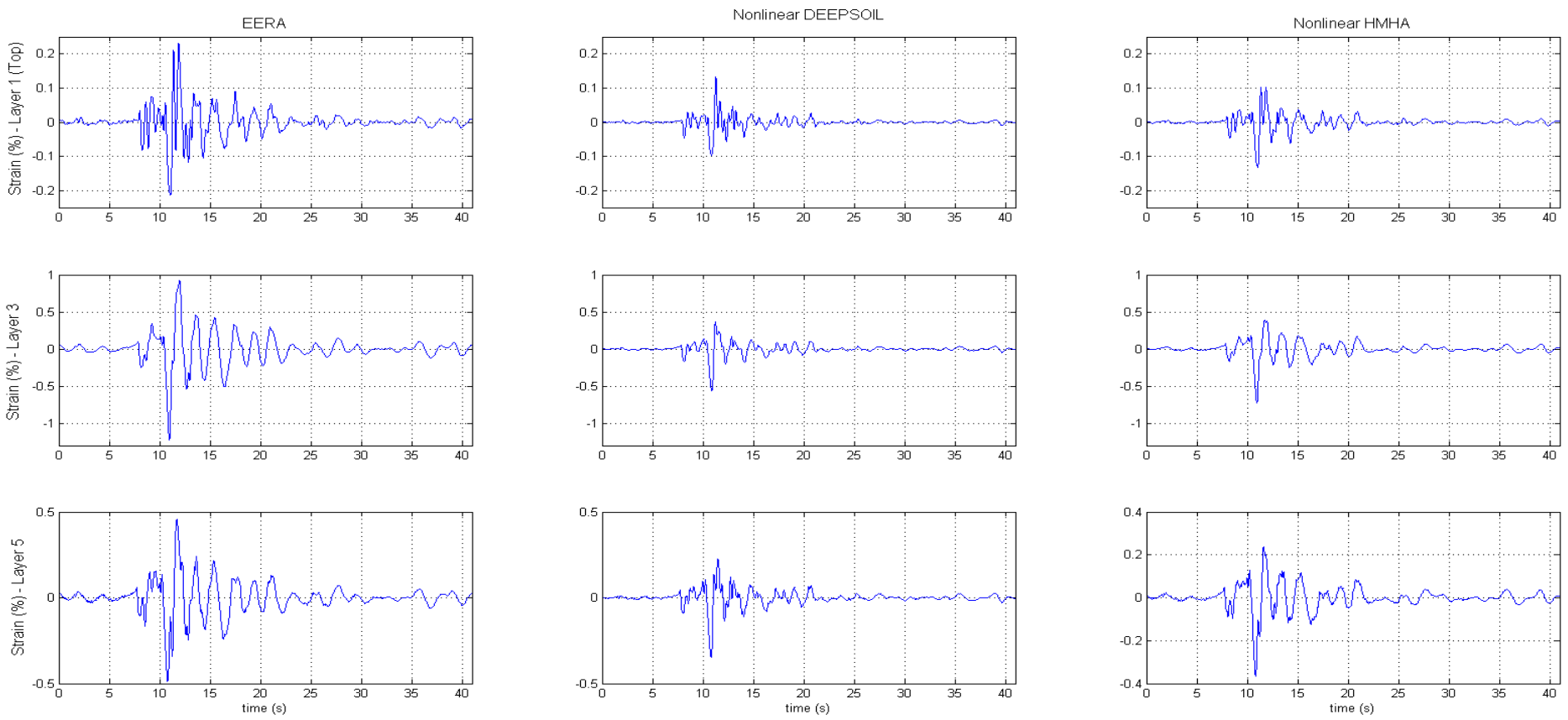

Fig. 3: Strain time history of layer 1, layer 3 and layer 5 for the EERA, DEEPSOIL and NHMHA.

Figure 2 illustrate the time evolution of the acceleration, at 3 different layers (1, 3 and 5) taking to account different methods, the Equivalent Linear Response Analysis (EERA), the Nonlinear Harmonic-Modal Hybrid Approach (NHMHA) 
proposed here and the Nonlinear method used in DEEPSOIL software. It can be observed that both nonlinear methods have a very similar behavior, unlike the equivalent linear method that presents certain differences, especially in Figure (3) where we can observe the strain time history, in which EERA results vary significantly. The main reason for this is because the equivalent linear analysis is common to characterize the level of a transient strain time history in terms of an effective shear strain, which has been empirically calculated and has a variation between $50 \%$ and $70 \%$ of the maximum shear strain.

The computational procedure when employing the parametric solution based on the PGD allowed reducing by more than one order of magnitude the online computing time, from one minute to a few seconds.

\section{Conclusion}

This paper proposes a new dynamic calculation of the nonlinear soil behavior based on a hybrid technique able to compute very fast solutions. The approach combines different ingredients: (i) modal analysis (ii) a harmonic space-frequency description of the dynamic problem; (iii) the introduction of material parameters as model extra-coordinates, (iv) an online integration that proceeds by particularizing the parametric solution for the damping parameters, and then updating the damping parameters, regard the level of deformation, from the just calculated solution. The results of this new approach were satisfactory, which allows us to avoid traditional methods based on the equivalent linear analysis in which an approximation to the non-linear behavior of the soil is obtained. Model parameters have been considered as extra-coordinates for constructing parametric solutions that can be seen as computational vademecums from which we can perform in real time, optimization, inverse analysis and simulation based control.

\section{Acknowledgements}

This research has been funded by National Fund for Innovation and Scientific and Technological Development (FONDOCYT). This support is gratefully acknowledged.

\section{References}

[1] Chinesta, F., Keunings, R., \& Leygue, A. (2013). The proper generalized decomposition for advanced numerical simulations: a primer. Springer Science \& Business Media.

[2] Chinesta, F., Ammar, A., \& Cueto, E. (2010). Recent advances and new challenges in the use of the proper generalized decomposition for solving multidimensional models. Archives of Computational methods in Engineering, 17(4), 327350 .

[3] Chinesta, F., Leygue, A., Bordeu, F., Aguado, J. V., Cueto, E., González, D., \& Huerta, A. (2013). PGD-based computational vademecum for efficient design, optimization and control. Archives of Computational Methods in Engineering, 20(1), 31-59.

[4] Clough, R. W., \& Penzien, J. (2003). Dynamics of structures. Berkeley. CA: Computers and Structures, Inc.

[5] Germoso, C., Aguado, J. V., Fraile, A., Alarcon, E., \& Chinesta, F. (2016). Efficient PGD-based dynamic calculation of non-linear soil behavior. Comptes Rendus Mecanique, 344(1), 24-41.

[6] Hardin, B.O. and Drnevich, V.P. (1972). "Shear Modulus and Damping in Soils: Design Equation and Curves." Proc. ASCE, Vol. 98, SM7, 667-692.

[7] Ishihara, K. (1982). "Evaluation of soil properties for use in earthquake response analysis." International Symposium on 9umerical Models in Geomechanics,Zurich.

[8] Kramer, S. L. (1996). Geotechnical earthquake engineering. Pearson Education India.

[9] Kondner, R. L., and Zelasko, J. S. (1963). "A hyperbolic stress-strain formulation of sands." Proc. 2nd Pan Am. Conf. on Soil Mech. And Found. Engrg., Brazilian Association of Soil Mechanics, Sao Paulo, Brizil, 289-324

[10] Lysmer, J., Kuhlemeyer, A.M. Finite dynamics model for infinite media, J. Eng. Mech. 95 (1969) 859-877.

[11] Malik, M. H., Borzacchiello, D., Aguado, J. V., \& Chinesta, F. (2018). Advanced parametric space-frequency separated representations in structural dynamics: A harmonic-modal hybrid approach. Comptes Rendus Mécanique, 346(7), 590602. 
[12] Pruliere, E., Chinesta, F., \& Ammar, A. (2010). On the deterministic solution of multidimensional parametric models using the proper generalized decomposition. Mathematics and Computers in Simulation, 81(4), 791-810.

[13] Quaranta, G., Martin, C. A., Ibáñez, R., Duval, J. L., Cueto, E., \& Chinesta, F. (2019). From linear to nonlinear PGDbased parametric structural dynamics. Comptes Rendus Mécanique, 347(5), 445-454. 